\title{
Inhibition of histone deacetylase 1 ameliorates renal tubulointerstitial fibrosis via modulation of inflammation and extracellular matrix gene transcription in mice
}

\author{
TÙNG NGUYỄN-THANH ${ }^{1-3}$, DAL KIM $^{1}$, SIK LEE $^{1,2}$, WON KIM $^{1,2}$, \\ SUNG KWANG PARK ${ }^{1,2}$ And KYUNG PYO KANG ${ }^{1,2}$ \\ ${ }^{1}$ Department of Internal Medicine, Chonbuk National University Medical School; \\ ${ }^{2}$ Research Institute of Clinical Medicine of Chonbuk National University-Biomedical Research Institute of \\ Chonbuk National University Hospital, Jeonju, Jeollabuk 54907, Republic of Korea
}

Received February 18, 2017; Accepted October 19, 2017

DOI: $10.3892 / \mathrm{ijmm} .2017 .3218$

\begin{abstract}
Renal tubulointerstitial fibrosis is characterized by sustained inflammation and excessive extracellular matrix (ECM) accumulation, leading to chronic kidney disease. Valproic acid (VPA) has anticancer activity through regulation of cell differentiation and apoptosis via inhibition of histone deacetylase (HDAC) activity and is considered a class I HDAC inhibitor. In this study, the effect of VPA on unilateral ureteral obstruction (UUO)-induced renal fibrosis by modulation of renal inflammation and ECM gene transcription was investigated. VPA treatment increased histone H3 acetylation in both shamand UUO-operated kidneys and decreased the UUO-induced increase in tubular injury and ECM deposition in mice. VPA also decreased myofibroblast activation and proliferation in UUO kidneys and NRK-49F cells. Finally, it was demonstrated that the anti-fibrotic effect of VPA was associated with regulation of ECM protein promoter enrichment at an acetylated histone $\mathrm{H} 3$ site. In conclusion, the findings indicate that VPA may have a beneficial effect on UUO-induced renal fibrosis via regulation of myofibroblast activation, proliferation, and ECM protein production by chromatin remodeling and ECM protein promoter transcription.
\end{abstract}

\section{Introduction}

Chronic kidney disease (CKD) is a global health burden that affects $>10 \%$ of adults worldwide (1). Despite advances in

Correspondence to: Professor Kyung Pyo Kang or Professor Sung Kwang Park, Department of Internal Medicine, Chonbuk National University Medical School, 20 Geonjiro, Deokjin-gu, Jeonju, Jeollabuk 54907, Republic of Korea

E-mail: kpkang@chonbuk.ac.kr

E-mail:parksk@chonbuk.ac.kr

Present address: ${ }^{3}$ Department of Histology and Embryology, Hue University of Medicine and Pharmacy, Hue City, Vietnam

Key words: histone deacetylase inhibitor, fibroblast, inflammation, kidney fibrosis, extracellular matrix therapeutic strategies, CKD is considered an independent risk factor for cardiovascular disease (2-4). Furthermore, CKD is associated with high morbidity and mortality in earlier stages (3). For patients with stage 5 on CKD on dialysis, the mortality rate is 10-100 times greater than that in an age-matched population with normal renal function (3). In terms of economics, CKD is associated with high healthcare cost and loss of productivity $(3,5)$. Therefore, optimal management of CKD is necessary for improvement of global public health problems.

Tubulointerstitial fibrosis is a common final feature of kidney disease, irrespective of glomerular, tubular and capillary injury (6). The fibrotic process is characterized by sustained inflammation, including inflammatory cell infiltration and secretion of cytokines, excessive extracellular matrix (ECM) accumulation and imbalance of ECM degradation, leading to organ dysfunction $(7,8)$. Myofibroblasts are key mediators of fibrosis that produce and secrete ECM following activation (6). This fibrotic process is mediated by transforming growth factor- $\beta$ (TGF- $\beta$ ) and its downstream signaling pathways, which have roles in cellular proliferation, differentiation and growth (9). Our previous study reported that tamoxifen, a selective estrogen receptor (ER) modulator, has a protective effect on renal fibrosis through suppression of TGF- $\beta$-induced renal fibroblast activation, ECM deposition and inflammation via modulation of ER $\alpha$-dependent TGF- $\beta /$ Smad signaling (10). Therefore, identifying for a novel therapeutic target to reverse renal fibrosis is a challenge for preventing and treating progressive CKD.

Valproic acid (VPA) is a branched short-chain fatty acid that is prescribed for treatment of seizure disorders, mood disorders and migraine headaches (11). The mechanism of action is associated with regulation of $\gamma$-amino butyrate neurotransmitter activity. Previously, VPA has been reported to have anticancer activity via regulation of cell differentiation and apoptosis by inhibition of histone deacetylase (HDAC) activity, and is considered a class I HDAC inhibitor $(12,13)$. HDACs function by removing the acetyl groups from acetylated histones and/or non-histone proteins to regulate gene transcription and protein functions (14). HDACs are expressed in the developing kidney, renal tubular cells and renal fibroblasts, 
and have been reported to be involved in cellular proliferation, survival, differentiation and immunological responses. Several studies have investigated the protective effect of VPA in a renal injury model, including diabetic nephropathy (15), adriamycin-induced glomerular sclerosis model (16) and sepsis-induced kidney injury (17). However, the effect of VPA on unilateral ureteral obstruction (UUO)-induced fibroblast activation, inflammation and ECM accumulation, and their mechanisms remain elusive. Therefore, the current study aimed to investigate whether inhibition of HDAC1 by VPA has a protective effect on UUO-induced renal fibrosis via modulation of renal inflammation and ECM gene transcription.

\section{Materials and methods}

Animal experiments. The animal experiment protocol was reviewed and approved by the Institutional Animal Care and Use Committee of Chonbuk National University (Jeonju, Korea). Male C57BL/6 mice ( $\mathrm{n}=60 ; 7$ weeks old; weighing 20-23 g) were purchased from Orient Bio, Inc. (Seoul, Korea) and maintained in a room under controlled temperature $\left(23 \pm 1^{\circ} \mathrm{C}\right)$, humidity, lighting ( $12 \mathrm{~h}$ light $/ 12 \mathrm{~h}$ dark cycle) and with free access to chow and water. For the experiment, the mice were divided into four groups: i) Sham with vehicle treatment; ii) UUO with vehicle treatment; iii) sham with VPA treatment; and iv) UUO with VPA treatment ( $\mathrm{n}=15$ each group). VPA (Sigma-Aldrich; Merck KGaA, Darmstadt, Germany) was dissolved in PBS. PBS was used as the vehicle. VPA $(300 \mathrm{mg} / \mathrm{kg})$ was administered by intraperitoneal injection once a day for 5 days prior to UUO surgery and continued for 14 days after UUO surgery.

Renal fibrosis was induced by UUO operation as described previously (10). In brief, mice were anesthetized via intraperitoneal injection of ketamine $(100 \mathrm{mg} / \mathrm{kg}$; Huons Co., Ltd., Seoul, Korea) and xylazine (10 mg/kg; Bayer, Newbury, UK) and placed on temperature-controlled operating table with body temperature maintained at $37^{\circ} \mathrm{C}$. Following midline incision in the abdomen, the right proximal ureteral was exposed and ligated at two separated points using 3-0 black silk. The sham operation was performed using the same method without ligation of the ureter. At 2 weeks after UUO, the obstructed kidney was harvested, prepared for histological examination, and stored at $-80^{\circ} \mathrm{C}$ for western blot analysis and cytokine assays.

Histological examination. The kidneys were fixed via immersion in $4 \%$ paraformaldehyde at $4^{\circ} \mathrm{C}$ for $24 \mathrm{~h}$, dehydrated by washing in a series of increasing ethanol concentrations (70, 95 and $100 \%)$ for $1 \mathrm{~h}$, and then embedded in paraffin. The block was cut into 5- $\mu \mathrm{m}$ sections and stained with $0.5 \%$ periodic acid-Schiff stain (PAS) for 15 min and Masson's trichrome (MTC) using biebrich scarlet-acid fuchsin solution and phosphomolybdic-phosphotungstic acid solution for $15 \mathrm{~min}$. Immunohistochemical and immunofluorescence staining was performed as described previously (10). For immunohistochemical staining, the following primary antibodies were used: goat anti-mouse type I collagen (1310-01; 1:100; SouthernBiotech, Birmingham, AL, USA), hamster anti-mouse intercellular adhesion molecule-1 (ICAM-1; 553249; 1:100; BD Biosciences, San Jose, CA, USA) or rabbit anti-mouse monocyte chemoattractant protein-1 (MCP-1; 70R50662;
1:100; Fitzgerald Industries International, Acton, MA, USA). For immunofluorescence staining, rabbit anti-fibroblast specific protein-1 (FSP-1) antibody (ab93283; 1:100; Abcam, Cambridge, UK), anti- $\alpha$-smooth muscle actin ( $\alpha$-SMA; BD Biosciences), rat anti-mouse Ki-67 (14-5698-82; 1:200; Thermo Fisher Scientific, Inc., Waltham, MA, USA) or rat anti-mouse F4/80 (14-4801-82; 1:200; eBioscience, San Diego, CA, USA) were used as primary antibodies and then FITC- or Cy3-labeled as secondary antibodies (Chemicon; Merck \& Co., Inc., Whitehouse Station, NJ, USA). The nuclear staining was performed using $300 \mathrm{nM}$ DAPI solution for $3 \mathrm{~min}$ (Molecular Probes; Thermo Fisher Scientific, Inc.). For morphometric analysis, two observers unaware of the origins of samples used a Zeiss Z1 microscope or Zeiss LSM 510 confocal microscope (Carl Zeiss AG, Oberkochen, Germany) to evaluate all slides. The tubular injury was scored at six levels on the basis of the percentage of tubular dilatation, epithelial desquamation and loss of brush border in 10 randomly chosen, non-overlapping fields at a magnification of $\mathrm{x} 200$ under a light microscope: 0 , none; $0.5,<10 \% ; 1,10$ to $25 \% ; 2,25$ to $50 \% ; 3,50$ to $75 \%$; and $4,>75 \%$. The fibrotic areas and areas positive for type I collagen, ICAM-1 and MCP-1 were measured in 10 randomly chosen, non-overlapping fields at a magnification of x200 using ImageJ software (http://rsb.info.nih.gov/ij). The number of Ki-67 and $\alpha$-SMA double-positive myofibroblasts and F4/80-positive macrophages was counted at a magnification of $\mathrm{x} 400$.

Picrosirius red stain. For evaluation of the collagen deposition after ureteral obstruction, paraffin-embedded tissue sections were stained with Picrosirius red (10). The Picrosirius red-positive areas were measured in 10 randomly chosen, non-overlapping fields at a magnification of x 200 using ImageJ software.

Western blotting. Western blot analysis was performed as described previously (10). Primary antibodies $\alpha$-SMA (A2547; mouse) and vimentin (V6630; mouse) (1:1000; Sigma-Aldrich; Merck KGaA), ICAM-1 (sc-1511; goat; 1:1,000), fibronectin (sc-6953; goat; 1:500), and Smad7 (sc-11392; rabbit; 1:1,000) (Santa Cruz Biotechnology, Inc., Dallas, TX, USA), type I collagen (1310-01; goat; 1:1,000; SouthernBiotech), phospho-Smad2 (3101; rabbit), phospho-Smad3 (9520; rabbit), acetyl-histone H3 (14932; rabbit), and histone H3 (9715; rabbit) (1:1,000; Cell Signaling Technology Inc., Danvers, MA, USA), and Smad2/3 (07-408; rabbit; 1:1,000; EMD Millipore, Billerica, MA, USA) were used. GAPDH (AP0063; rabbit; 1:2,000; Bioworld Technology, Inc., St. Louis Park, MN, USA) was used as an internal control. All signals were analyzed by densitometric scanning (LAS-3000; FujiFilm, Tokyo, Japan).

Measurement of renal MCP-1 and TGF- $\beta 1$ levels. The MCP-1 (MJE00) and TGF- $\beta 1$ (MB100B) levels were determined by ELISA kits (R\&D Systems, Inc., Minneapolis, MN, USA) according to the manufacturer's instructions.

Cell culture experiment. In vitro experiments were performed using rat renal fibroblast cell line (NRK-49F; American Type Culture Collection, Manassas, VA, USA). NRK-49F cells were cultured in Dulbecco's modified Eagle's medium with $4 \mathrm{mM}$ L-glutamine adjusted to contain $1.5 \mathrm{~g} / 1$ sodium bicarbonate and $4.5 \mathrm{~g} / \mathrm{l}$ glucose supplemented with $5 \%$ (vol/vol) 
heat-inactivated fetal bovine serum and antibiotics (100 U/ $\mathrm{ml}$ penicillin $\mathrm{G}$ and $100 \mu \mathrm{g} / \mathrm{ml}$ streptomycin) at $37^{\circ} \mathrm{C}$ with $5 \% \mathrm{CO}_{2}$ in $95 \%$ air. To investigate the effect of VPA on myofibroblast activation and ECM protein expression, subconfluent NRK-49F cells were incubated with VPA $(0.1,1$ and $2.5 \mathrm{mM}$ ) for $30 \mathrm{~min}$ and then stimulated with TGF- $\beta 1$ (2 ng/ $\mathrm{ml}$; Sigma-Aldrich; Merck KGaA) for $24 \mathrm{~h}$. To examine the effects of VPA on HDAC1-mediated ECM protein expression, HDAC1 small interfering RNA (siRNA; 25 pmol; sc-270070; Santa Cruz Biotechnology, Inc.) or non-target control siRNA (25 pmol; sc-37007; Santa Cruz Biotechnology, Inc.) were tranfected using Lipofectamine ${ }^{\circledR} 2000$ (Invitrogen; Thermo Fisher Scientific, Inc.) in subconfluent NRK49F cells. Following knockdown of HDAC1 for $24 \mathrm{~h}$, cells were incubated with VPA $(2.5 \mathrm{mM})$ for $30 \mathrm{~min}$ and then stimulated with TGF- $\beta 1$ $(2 \mathrm{ng} / \mathrm{ml})$ for $24 \mathrm{~h}$.

Cell proliferation assay. After $24 \mathrm{~h}$ treatment with VPA $(0.1,1$ and $2.5 \mathrm{mM})$ and TGF- $\beta 1(2 \mathrm{ng} / \mathrm{ml})$, proliferation of NRK-49F cells was determined by a colorimetric assay (Cell Proliferation kit II; Roche Diagnostics GmbH, Mannheim, Germany) according to the manufacturer's protocol. All experimental values were determined from triplicate wells.

Wound healing assay. Subconfluent NRK-49F cells were cultured in 6-well dishes. Prior to treatment with VPA and TGF- $\beta 1$, dishes were scratched using a sterile $200 \mu$ l pipette tip, causing three separate wounds. The cells were incubated with VPA $(2.5 \mathrm{mM})$ for $30 \mathrm{~min}$ and then stimulated with TGF- $\beta 1$ ( $2 \mathrm{ng} / \mathrm{ml})$ for $24 \mathrm{~h}$. Wound lengths were measured using the ImageJ program. At $0 \mathrm{~h}$ after scratching, this wound length was used as the control.

Chromatin immunoprecipitation (ChIP) assay. ChIP assay was performed using the acetyl-histone H3 immunoprecipitation kit (17-245; EMD Millipore) according to the manufacturer's protocol. In brief, subconfluent NRK-49F cells were incubated with VPA $(2.5 \mathrm{mM})$ for $30 \mathrm{~min}$ and then stimulated with TGF- $\beta 1(2 \mathrm{ng} / \mathrm{ml})$ for $1 \mathrm{~h}$. For cross-linking histones to DNA, cells were treated with $1 \%$ formaldehyde for $10 \mathrm{~min}$ at $37^{\circ} \mathrm{C}$. After washing and harvesting the cells, genomic DNA fragments were obtained by sonicating cell lysates. The cross-linked histone-DNA complexes were immunoprecipitated using antibody against acetyl-histone $\mathrm{H} 3$ and normal rabbit IgG as a negative control. Following precipitation with salmon sperm DNA/protein A agarose slurry, the histone-DNA complexes were eluted and reversed by heating at $65^{\circ} \mathrm{C}$ for $4 \mathrm{~h}$. The DNA was recovered by phenol/chloroform extraction. The inputs consisted of $5 \%$ chromatin before immunoprecipitation. The ChIP-enriched DNA and input DNA were analyzed by quantitative real time polymerase chain reaction (qRT-PCR) using the following primers: Fibronectin $1(F n l)$ promoter forward, 5'-CGTACCCTGGAAAGTC-3' and reverse, 5'-CTAAGCCT ACCTAACACCGA-3'; type I collagen $\alpha 1$ (Coll $\alpha 1)$ promoter forward, 5'-GCAGACTCTTCTAGCCGCTG-3' and reverse, 5'-CTATGTCGGCAGACAGGCTC-3'. qRT-PCR was performed using a SYBR-Green PCR Master Mix (Applied Biosystems, Carlsbad, CA, USA) on a rotor-gene Q 2plex system (Qiagen, Hilden, Germany) to measure ChIP-enriched
DNA and input DNA expression. The PCR program was as follows: $2 \mathrm{~min}$ at $50^{\circ} \mathrm{C}, 10 \mathrm{~min}$ at $95^{\circ} \mathrm{C}$, then $95^{\circ} \mathrm{C}$ for $15 \mathrm{sec}$, and $60^{\circ} \mathrm{C}$ for $1 \mathrm{~min}$ for 40 cycles. The relative expression of specific promotor sequences in immunoprecipitated DNA was determined using the $\Delta \Delta \mathrm{Ct}$ method. The relative enrichment of promoter DNA was normalized to the input.

Statistical analysis. Data are expressed as mean \pm standard deviation. Multiple comparisons were examined for significant differences using analysis of variance, followed by individual comparison with the Tukey post hoc test. $\mathrm{P}<0.05$ was considered to indicate a statistically significant difference.

\section{Results}

VPA decreases UUO-induced renal tubular injury and fibrosis. VPA has been reported as a class I HDAC inhibitor, therefore the effect of VPA on histone $\mathrm{H} 3$ acetylation (H3Ac) following UUO surgery. In sham-operated kidneys, VPA treatment increased H3Ac at lysine 9 and 14 compared with the vehicle-treated group. UUO kidneys from VPA-treated mice exhibited an increase in H3Ac compared to vehicle-treated UUO mice. These data suggest that VPA treatment significantly increases H3Ac in both sham and UUO kidneys (Fig. 1A).

To investigate the effect of VPA on UUO-induced renal tubulointerstitial fibrosis, kidney sections were examined following PAS and MTC staining. After 2 weeks of ureteral obstruction, UUO kidneys from vehicle-treated mice exhibited an increase in tubular dilatation, inflammatory cells and fibroblast-like cell infiltration, and tubulointerstitial fibrosis compared with sham-operated kidneys treated with vehicle or VPA. The VPA-treated UUO kidneys exhibited relatively preserved normal tubular structures and significantly reduced tubular injury, tubulointerstitial inflammation and fibrosis (Fig. 1B-D). These data suggest that VPA ameliorates UUO-induced tubular injury and fibrosis in vivo.

VPA reduces UUO-induced renal interstitial fibroblast activation and proliferation. Renal interstitial fibroblast activation is one mechanism of renal fibrogenesis (18). Therefore, we evaluated renal interstitial fibroblast expression after ureteral obstruction using anti-FSP-1 antibody. At 2 weeks after ureteral obstruction, the number of FSP-1-positive fibroblasts was increased in the tubulointerstitial area compared with sham-operated kidneys. VPA treatment significantly decreased the number of FSP-1-positive fibroblasts in UUO kidneys (Fig. 2).

To address the effect of VPA on UUO-induced myofibroblast activation and proliferation, double-positive $\alpha$-SMA and Ki-67 myofibroblast expression was evaluated following ureteral obstruction. The number of $\alpha$-SMA- and Ki-67-double positive myofibroblasts was increased in the tubulointerstitial area compared with sham-operated kidneys. VPA treatment mitigated the myofibroblast proliferation and infiltration in UUO kidneys (Fig. 3A). Protein expression of $\alpha$-SMA and vimentin was significantly increased in UUO kidneys from vehicle-treated mice compared with sham-operated kidneys. UUO kidneys from VPA-treated mice exhibited decreased $\alpha$-SMA and vimentin expression of $\sim 39.8$ and $67.5 \%$, respectively, compared with the UUO kidneys from vehicle-treated 

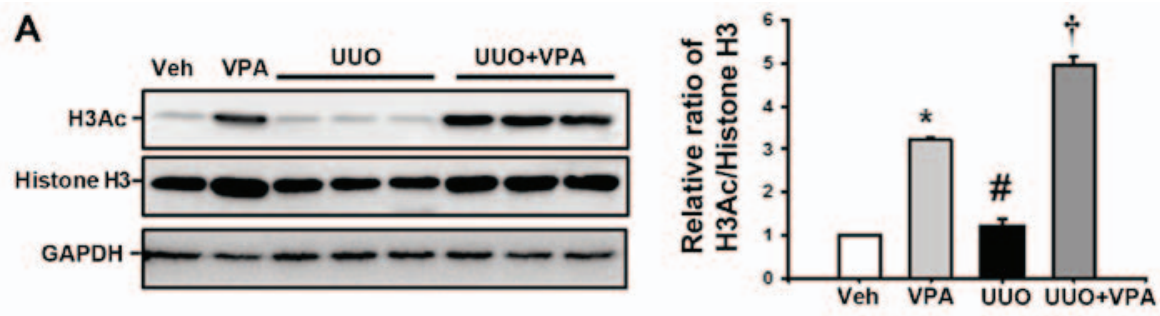

B

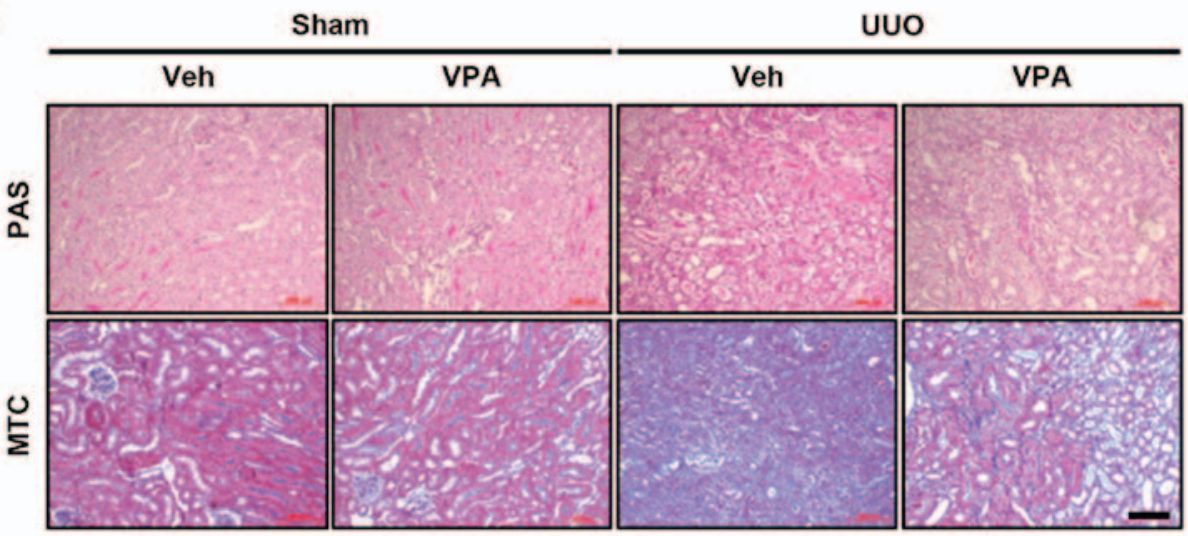

C
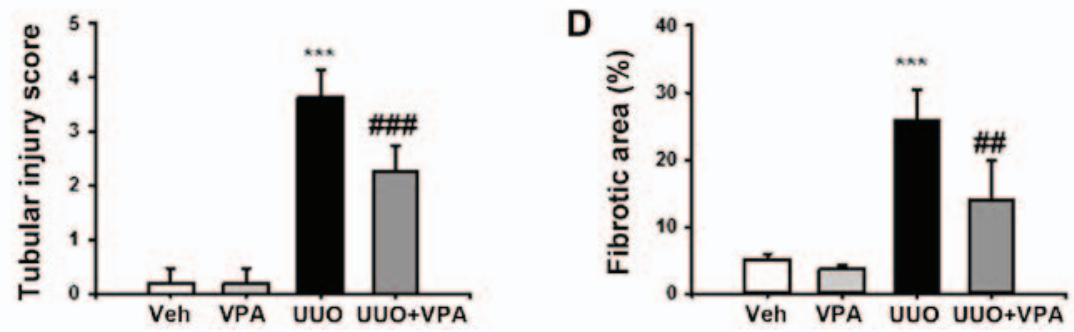

Figure 1. Effect of VPA on UUO-induced renal tubular injury and fibrosis. (A) Representative western blot analysis of H3Ac at lysine 9 and 14 from shamand UUO-operated mice treated with Veh or VPA. Data from densitometric analyses are presented as the relative ratio of each protein to histone H3. The relative ratio measured in the kidneys from sham-operated mice treated with Veh is arbitrarily presented as 1 . Data are expressed as the mean \pm SD of 3 independent experiments. "P $<0.05$ vs. Veh; ${ }^{~} \mathrm{P}<0.05$ vs. VPA; ${ }^{\prime P}<0.05$ vs. UUO. (B) Representative PAS and MTC-stained sections of kidneys from sham- and UUO-operated mice treated with Veh or VPA. Scale bar, $100 \mu \mathrm{m}$. Bar graphs show the semi-quantitative scoring of (C) tubular injury stained by PAS and (D) the area fractions (\%) of tubulointerstitial fibrosis stained by MTC in the sham and UUO-operated kidneys. Randomly chosen, non-overlapping fields $(\mathrm{n}=10)$ at a magnification of $\mathrm{x} 200$ were quantified ( $\mathrm{n}=15 /$ each group). Data are expressed as the mean $\pm \mathrm{SD}$ of 3 independent experiments. ${ }^{* * *} \mathrm{P}<0.001 \mathrm{vs}$. Veh or VPA; ${ }^{\# \#} \mathrm{P}<0.01$ vs. UUO; ${ }^{\# \#} \mathrm{P}<0.001$ vs. UUO. Veh, vehicle; VPA, valproic acid; Sham, sham-operated mice; UUO, unilateral ureteral obstruction-operated mice; H3Ac, acetylated histone 3; PAS, periodic acid-Schiff stain; MTC, Masson's trichrome; SD, standard deviation.
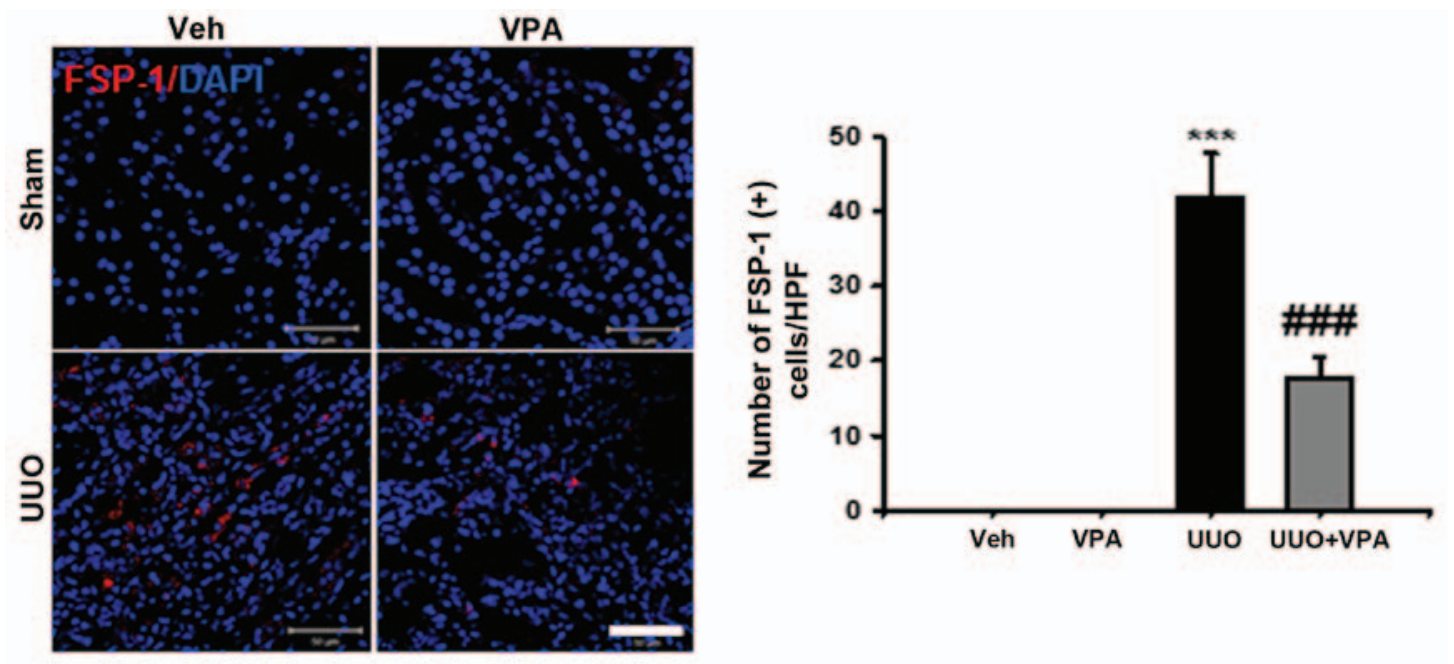

Figure 2. Effect of VPA on UUO-induced renal interstitial fibroblast activation. Representative immunofluorescence staining of FSP-1 from kidneys of sham- and UUO-operated mice treated with Veh or VPA. The nuclei were stained with DAPI. The bar graph shows the number of FSP-1-positive cells from ten randomly chosen, non-overlapping fields at a magnification of $x 400$ ( $\mathrm{n}=15 /$ each groups). Bar, $50 \mu \mathrm{m}$. Data are expressed as the mean \pm SD of 3 independent experiments. ${ }^{* * *} \mathrm{P}<0.001$ vs. Veh or VPA; ${ }^{\# \#} \mathrm{P}<0.001$ vs. UUO. Veh, vehicle; VPA, valproic acid; Sham, sham-operated mice; UUO, unilateral ureteral obstruction-operated mice; FSP-1, fibroblast-specific protein-1; SD, standard deviation. 
A
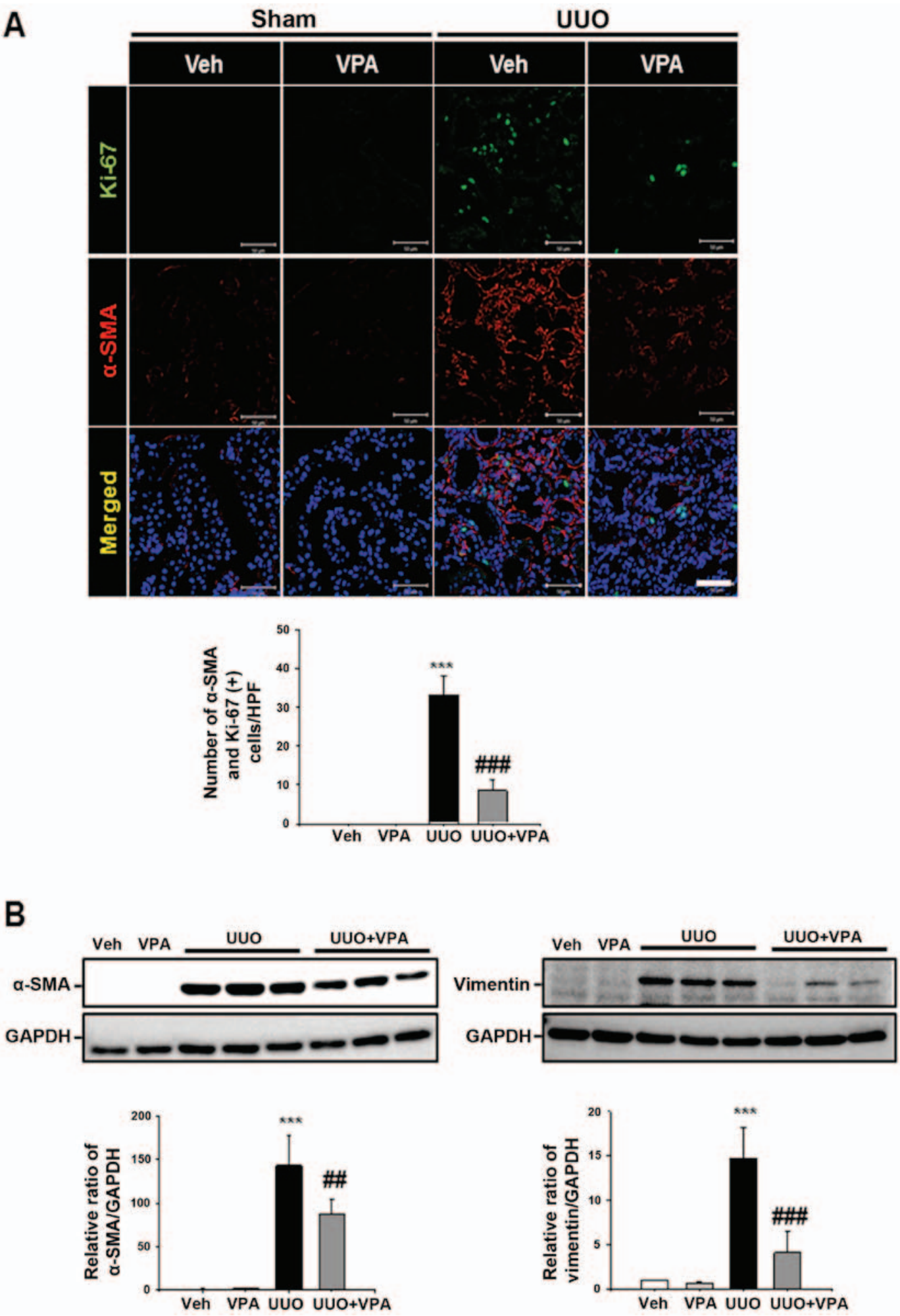

Figure 3. Effect of VPA on UUO-induced renal myofibroblast proliferation. (A) Representative double immunofluorescence staining of $\alpha$-SMA (red) and Ki-67 (green) from kidneys of sham- and UUO-operated mice treated with Veh or VPA. The nucleus was stained by DAPI. The bar graph shows the number of $\alpha$-SMA and Ki-67 double-positive cells from ten randomly chosen, non-overlapping fields at a magnification of $\mathrm{x} 400$ ( $\mathrm{n}=15 / \mathrm{each}$ groups). Data are expressed as the mean $\pm \mathrm{SD}$. Bar, $50 \mu \mathrm{m}$. (B) Representative western blot analysis of $\alpha$-SMA and vimentin from sham- and UUO-operated mice treated with Veh or VPA. Data from densitometric analyses are presented as the relative ratio of each protein to GAPDH. The relative ratio measured in the kidneys from sham-operated mice treated with Veh is arbitrarily presented as 1 . Data are expressed as the mean \pm SD of 3 independent experiments. ${ }^{* * *} \mathrm{P}<0.001 \mathrm{vs}$. Veh or VPA; ${ }^{\# / \prime} \mathrm{P}<0.01$

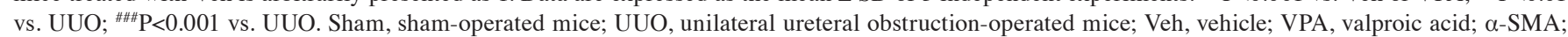
$\alpha$-smooth muscle actin; HPF, high power field; SD, standard deviation.

mice (Fig. 3B). These data suggest that VPA treatment mitigates UUO-induced renal myofibroblast activation and proliferation.
VPA decreases UUO-induced fibronectin and type I collagen expression. ECM deposition is an important process during renal fibrogenesis (18). Whether VPA regulates ECM 
A

A Sham

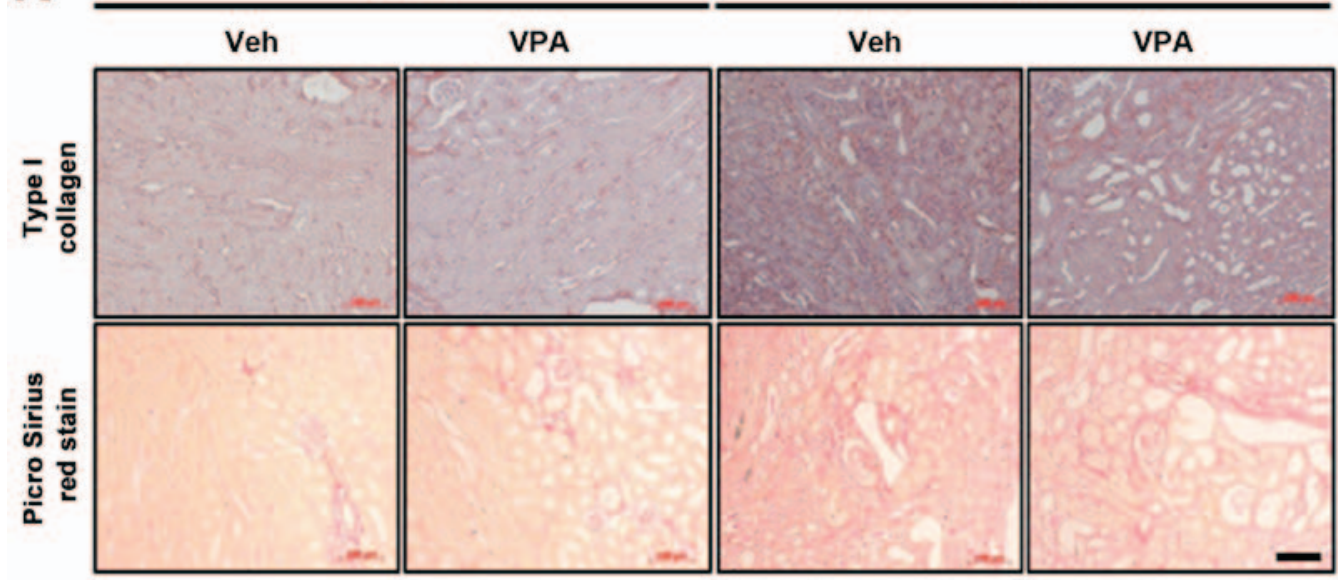

B

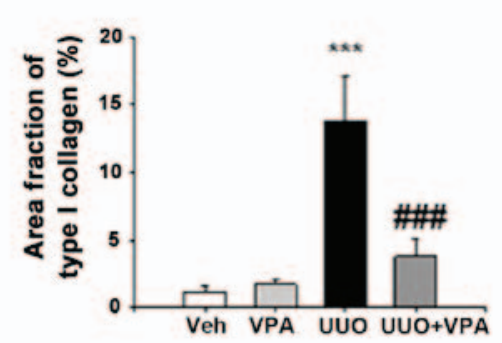

D

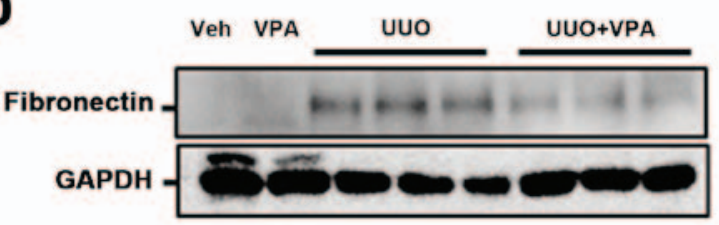

C
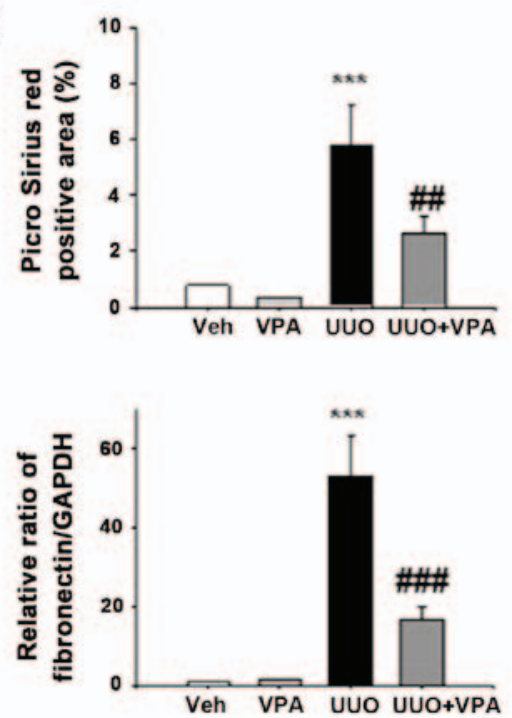

Figure 4. Effect of VPA on UUO-induced extracellular matrix deposition. (A) Representative immunohistochemistry of type I collagen and Picrosirius red staining from kidneys of sham- and UUO-operated mice treated with Veh or VPA. Bar, $100 \mu \mathrm{m}$. (B) The bar graph presents the area fractions (\%) of type I collagen from 10 randomly chosen, non-overlapping fields at magnification of $x 200$ ( $n=15 /$ each group). Data are expressed as the mean \pm SD. (C) The bar graph shows the area fractions (\%) of Picrosirius red staining from ten randomly chosen, non-overlapping fields at magnification of x200 (n=15/each groups). Data are expressed as mean \pm SD. (D) Representative western blot analysis of fibronectin from sham- and UUO-operated mice treated with Veh or VPA. Data from densitometric analyses are presented as the relative ratio of each protein to GAPDH. The relative ratio measured in the kidneys from sham-operated mice treated with Veh is arbitrarily presented as 1 . Data are expressed as the mean \pm SD of 3 independent experiments. ${ }^{* * *} \mathrm{P}<0.001$ vs. Veh or VPA; ${ }^{\# \# P} \mathrm{P}<0.01$ vs. UUO; ${ }^{\# \# \# P<0.001 ~ v s . ~ U U O . ~}$ Sham, sham-operated mice; UUO, unilateral ureteral obstruction-operated mice; Veh, vehicle; VPA, valproic acid; SD, standard deviation.

deposition following ureteral obstruction was evaluated in the current study. In immunohistochemistry analysis, UUO kidneys exhibited an increase in type I collagen expression in the tubulointerstitial area compared with sham-operated kidneys. Collagen fibril deposition was also evaluated by Picrosirius red stain. Deposition of collagen fibrils was significantly increased in the tubulointerstitial areas of UUO kidneys from vehicle-treated mice. VPA treatment significantly decreased the UUO-induced increase in type I collagen expression. UUO kidneys from VPA-treated mice exhibited a decrease in Picrosirius red-positive areas of $\sim 55.1 \%$ compared with UUO kidneys from vehicle-treated mice (Fig. 4A-C). In western blot analysis, fibronectin expression following ureteral obstruction was significantly increased compared to that in sham-operated kidneys. However, VPA treatment significantly decreased the UUO-induced increase of fibronectin expression by $\sim 68.4 \%$ (Fig. 4D). These data suggest that VPA ameliorates the UUO-induced increase in ECM deposition.
VPA ameliorates UUO-induced renal tubulointerstitial inflammation. Inflammation promotes progressive renal fibrosis (19). Therefore, the effects of VPA on macrophage infiltration, cell adhesion molecules and chemoattractant factor expression were examined following ureteral obstruction. The number of F4/80-positive macrophages was significantly increased in the tubulointerstitial areas in the UUO kidney compared with sham-operated kidneys (Fig. 5A). VPA treatment significantly reduced $\mathrm{F} 4 / 80$-positive macrophage infiltration in UUO kidneys compared with vehicle-treated UUO kidneys.

For evaluation of cell adhesion molecules and chemoattractant factor expression following ureteral obstruction, ICAM-1 and MCP-1 expression was assessed in UUO kidneys via immunohistochemistry. The expression of ICAM-1 was significantly increased in the interstitial area from UUO kidneys. VPA treatment significantly decreased the expression of ICAM-1 compared with vehicle-treated UUO kidneys (Fig. 5B). ICAM-1 protein expression in was also examined in kidney lysates via western 
A
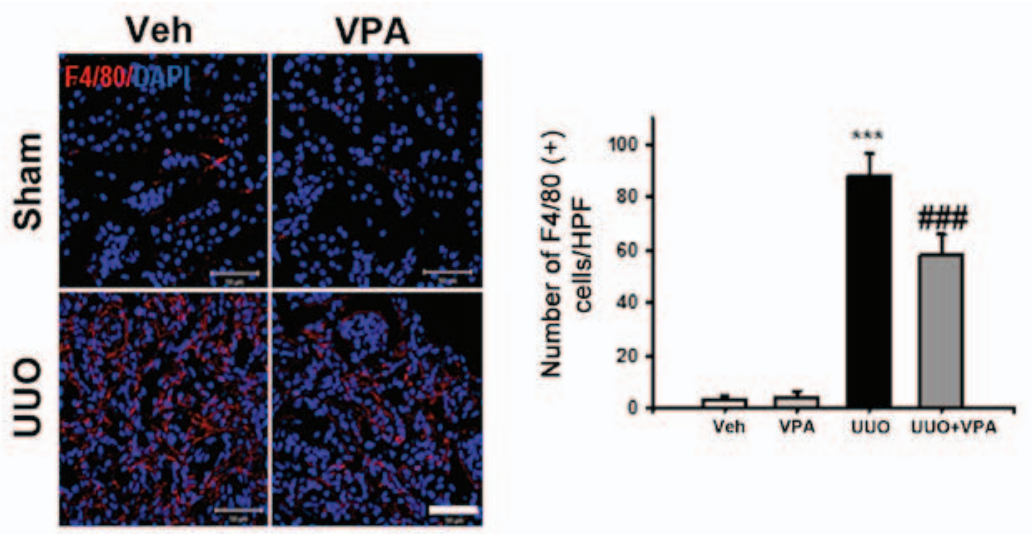

B

Sham

UUO
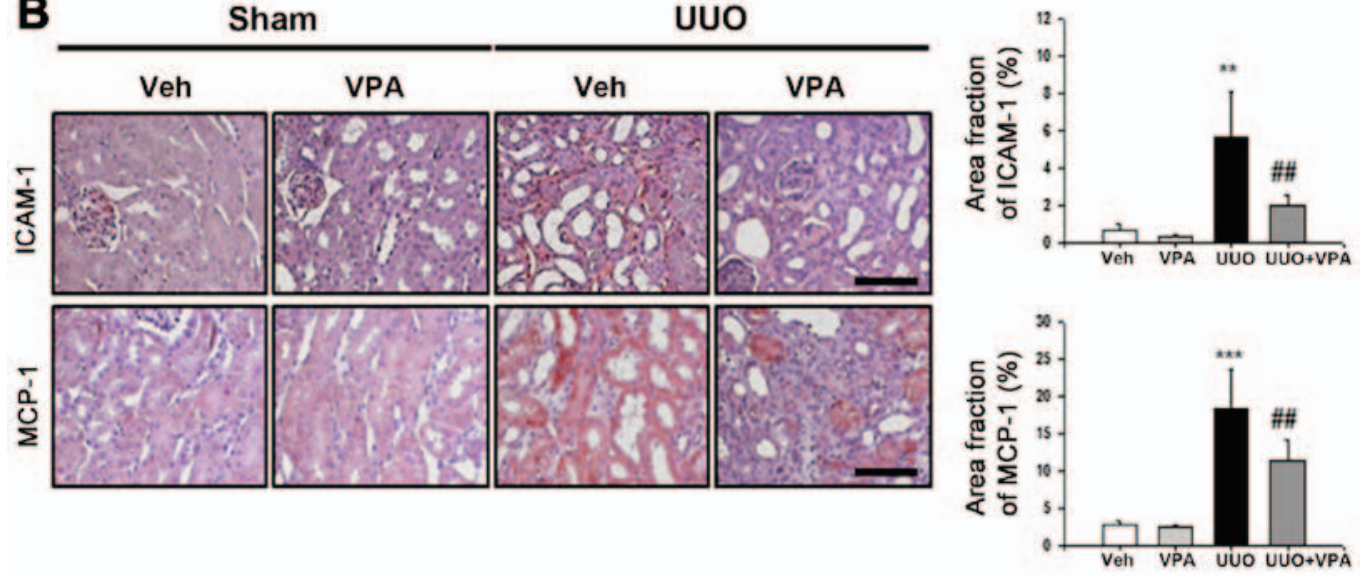

C
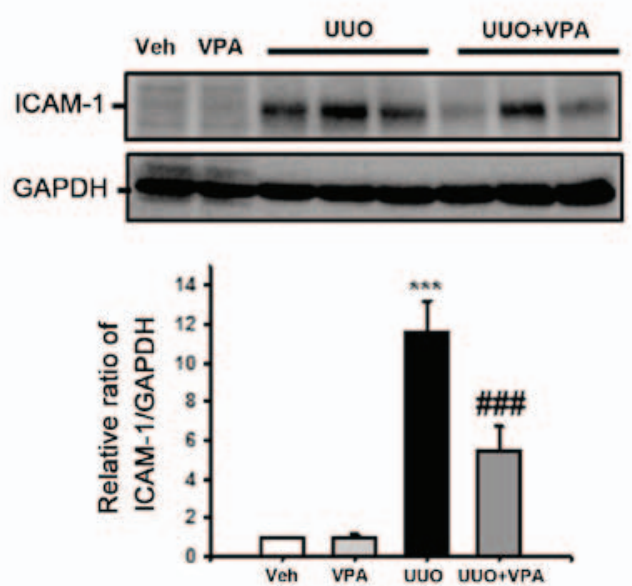

D

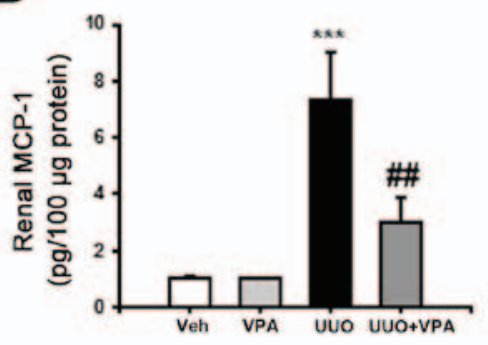

Figure 5. Effect of VPA on UUO-induced renal inflammation. (A) Representative immunofluorescence staining of F4/80-positive macrophages from kidneys of sham- and UUO-operated mice treated with Veh or VPA. The nucleus was stained by DAPI. The bar graph presents the number of F4/80-positive macrophages from 10 randomly chosen, non-overlapping fields at a magnification of $\mathrm{x} 400$ ( $\mathrm{n}=15 /$ each groups). Bar, $50 \mu \mathrm{m}$. Data are expressed as the mean $\pm \mathrm{SD}$. (B) Representative immunohistochemistry of ICAM-1 and MCP-1 staining from kidneys of sham- and UUO-operated mice treated with Veh or VPA. Bar, $100 \mu \mathrm{m}$. The bar graph shows the area fractions (\%) of ICAM-1 and MCP-1 from ten randomly chosen, non-overlapping fields at magnification of x200 ( $\mathrm{n}=15 /$ each groups). Data are expressed as the mean $\pm \mathrm{SD}$. (C) Representative western blot analysis of ICAM-1 from sham- and UUO-operated mice treated with Veh or VPA. Data from densitometric analyses are presented as the relative ratio of each protein to GAPDH. The relative ratio measured in the kidneys from sham-operated mice treated with Veh is arbitrarily presented as 1. Data are expressed as mean \pm SD of 3 independent experiments. (D) Renal MCP-1 levels from sham- and UUO-operated mice treated with Veh or VPA were measured by ELISA. The levels were normalized to $100 \mu \mathrm{g}$ of kidney protein. Data are expressed as the mean $\pm \mathrm{SD}$ of 3 independent experiments. ${ }^{* * *} \mathrm{P}<0.001$ vs. Veh or VPA; ${ }^{* *} \mathrm{P}<0.01$ vs. Veh or VPA; ${ }^{\# \#} \mathrm{P}<0.01$ vs. UUO; ${ }^{\# \# \#} \mathrm{P}<0.001$ vs. UUO Sham, sham-operated mice; UUO, unilateral ureteral obstruction-operated mice; Veh, vehicle; VPA, valproic acid; ICAM-1, intercellular adhesion molecule-1; MCP-1, macrophage chemoattractant protein-1; SD, standard deviation.

blot analysis. Similar to the findings from immunohistochemistry analysis, there was a significant decrease in the UUO-induced increase in ICAM-1 expression by $\sim 52.1 \%$ following VPA treatment (Fig. 5C). The expression of MCP-1 was significantly increased in the dilated tubules of UUO kidney compared with sham-operated kidney. VPA treatment significantly reduced the 
A
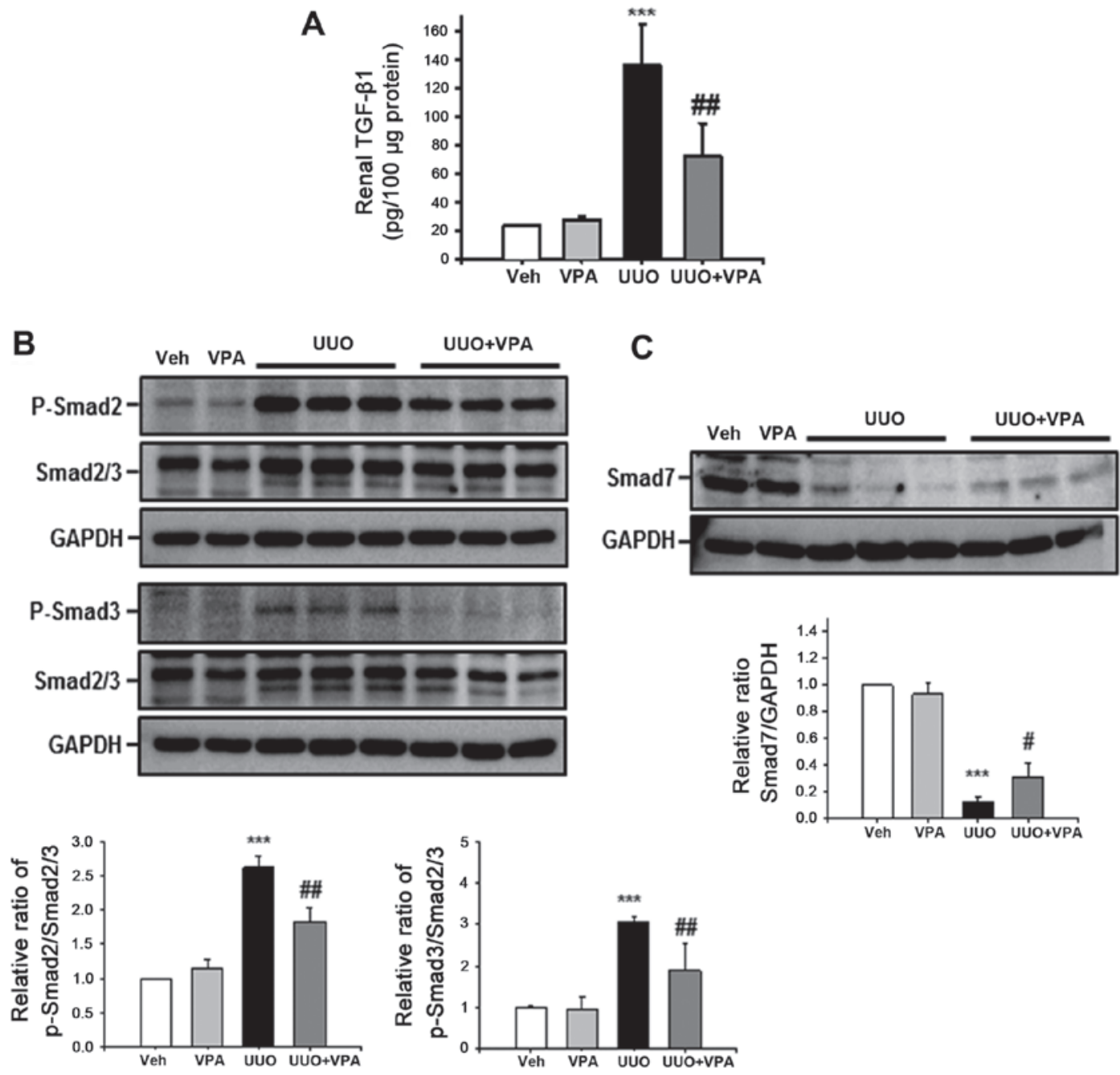

Figure 6. Effect of VPA on UUO-induced TGF- $\beta 1 /$ Smad signaling pathway. (A) TGF- $\beta 1$ levels in kidney tissue from sham- and UUO-operated mice treated with Veh or VPA were measured by ELISA. The levels were normalized to $100 \mu \mathrm{g}$ of kidney protein. Data are expressed as the mean \pm SD of 3 independent experiments. (n=5/each group). (B) Representative western blot analysis of P-Smad2 and P-Smad3 from sham- and UUO-operated mice treated with Veh or VPA. Data from densitometric analyses are presented as the relative ratio of each protein to Smad2/3. The relative ratio measured in the kidneys from sham-operated mice treated with Veh is arbitrarily presented as 1 . Data are expressed as the mean \pm SD of 3 independent experiments. (C) Representative western blot analysis of Smad7 from sham- and UUO-operated mice treated with Veh or VPA. Data from densitometric analyses are presented as the relative ratio of each protein to GAPDH. The relative ratio measured in the kidneys from sham-operated mice treated with Veh is arbitrarily presented as 1 . Data are expressed as the mean \pm SD of 3 independent experiments. ${ }^{* * *} \mathrm{P}<0.001$ vs. Veh or VPA; ${ }^{\#} \mathrm{P}<0.05$ vs. UUO; ${ }^{\# \#} \mathrm{P}<0.01$ vs. UUO; ${ }^{\# \#} \mathrm{P}<0.001$ vs. UUO. TGF- $\beta 1$, transforming growth factor- $\beta 1$; Veh, vehicle; VPA, valproic acid; P-Smad, phospho-Smad; UUO, unilateral ureteral obstruction-operated mice; SD, standard deviation.

UUO-induced increase in MCP-1 expression (Fig. 5B). Tissue MCP-1 protein levels in the UUO kidney were also evaluated by ELISA. The level of MCP-1 was significantly increased in UUO kidneys $(7.35 \pm 1.67 \mathrm{pg} / 100 \mu \mathrm{g}$ protein) compared with the level in vehicle-treated kidneys $(1 \pm 0.12 \mathrm{pg} / 100 \mu \mathrm{g}$ protein) or VPA-treated sham mice $(1.04 \pm 0.02 \mathrm{pg} / 100 \mu \mathrm{g}$ protein; Fig. 5D). VPA treatment reduced the UUO-induced increase in MCP-1 level (3.0 $\pm 0.88 \mathrm{pg} / 100 \mu \mathrm{g}$ protein). These data suggest that VPA reduces UUO-induced renal inflammation by regulation of macrophage infiltration and proinflammatory cytokine expression.

VPA regulates UUO-induced activation of TGF- $\beta 1 / \mathrm{Smad}$ signaling. Among pro-fibrogenic cytokines, TGF- $\beta 1$ is the major cytokine that drives renal fibrosis (20). To address the effect of VPA on the UUO-induced increase in TGF- $\beta 1$ expression, tissue TGF- $\beta 1$ level was evaluated using ELISA. The tissue TGF- $\beta 1$ level following ureteral obstruction was significantly increased $(135.4 \pm 29.2 \mathrm{pg} / 100 \mu \mathrm{g}$ protein) compared with vehicle- $(23.5 \pm 1.11 \mathrm{pg} / 100 \mu \mathrm{g}$ protein) and VPA- (28.2 $\pm 1.6 \mathrm{pg} / 100 \mu \mathrm{g}$ protein) treated sham mice (Fig. 6A). However, VPA treatment significantly reduced the UUO-induced increase in TGF- $\beta 1$ level $(72.7 \pm 22.5 \mathrm{pg} / 100 \mu \mathrm{g}$ protein). Phosphorylation of Smad 2 and Smad3 mediates downstream TGF- $\beta 1$ signaling, and protein binding to Smad-binding elements in DNA promotes profibrotic gene transcription (21). The UUO kidney from vehicle-treated mice had increased phosphorylation of Smad2 and Smad3 compared with sham-operated kidney (Fig. 6B). VPA-treated mice exhibited a significant reduction in the UUO-induced increase in $\mathrm{Smad} 2$ and Smad3 phosphorylation compared with vehicle-treated mice. By contrast, expression of inhibitory Smad7 was significantly decreased following ureteral obstruction (Fig. 6C). Smad7 expression was increased in UUO kidneys from VPA-treated mice compared with the UUO kidneys from vehicle-treated mice. These data suggest that VPA treatment regulates the UUO-induced TGF- $\beta 1 /$ Smad signaling pathway in vivo. 


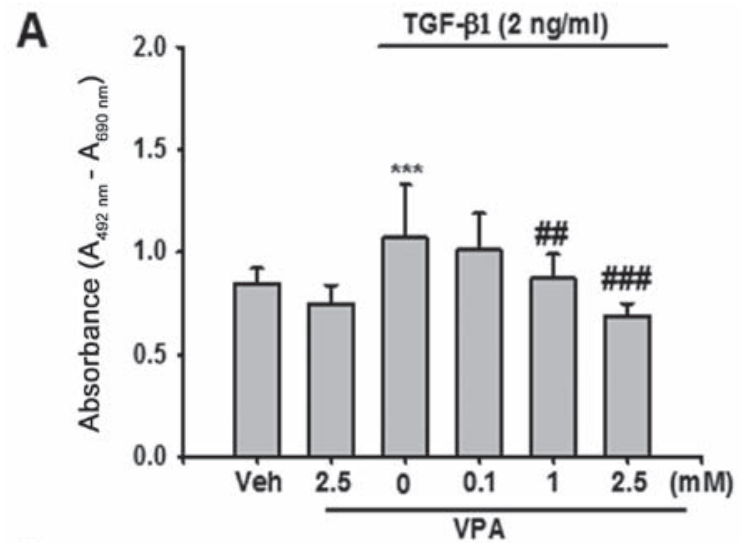

B

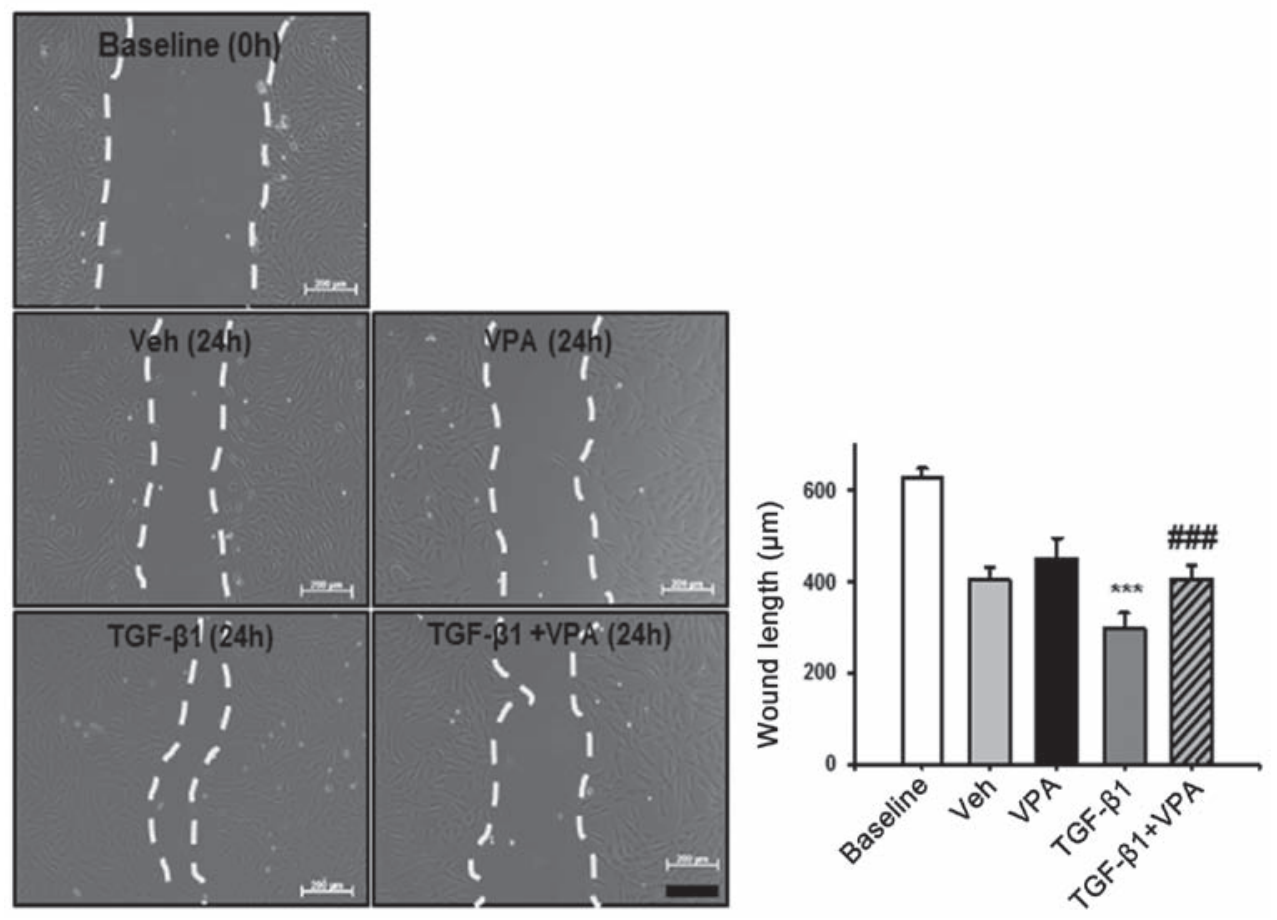

Figure 7. Effect of VPA on TGF- $\beta 1$-induced renal fibroblast proliferation and cell migration. (A) NRK-49F cells (2x10 3 ) were seeded in each well of 96 -well plates and treated with Veh or TGF- $\beta 1$ with or without VPA at indicated doses. After 24 h of treatment, cell proliferation was measured by XTT assay. Data are expressed as the mean \pm standard deviation for three independent experiments performed in triplicate. (B) Representative phase contrast images of NRK-49F cells after wound healing assay. The phase contrast images for NRK-49F cells that migrates into a scratch area obtained after either treatment with Veh, or TGF- $\beta 1(2 \mathrm{ng} / \mathrm{ml})$ with or without VPA $(2.5 \mu \mathrm{M})$ at 0 and $24 \mathrm{~h}$ after wound creation. The bar graph shows the mean length by which the gap between the NRK-49F cells closed over 0 or $24 \mathrm{~h}$ after treatment of Veh, TGF- $\beta 1$ and/or VPA. ${ }^{* * *} \mathrm{P}<0.001$ vs. Veh or VPA; ${ }^{\# \#} \mathrm{P}<0.001$ vs. TGF- $\beta 1{ }^{\# \# \#} \mathrm{P}<0.001$ vs. TGF- $\beta 1$. Bar, $200 \mu \mathrm{m}$. Veh, vehicle; VPA, valproic acid; TGF- $\beta 1$, transforming growth factor- $\beta 1$.

VPA decreases TGF- $\beta 1$-induced viability and migration of NRK-49F cells. To determine the protective mechanism of VPA in UUO-induced renal fibrosis, the effect of TGF- $\beta 1$ on renal interstitial fibroblast viability and migration was evaluated in vitro using NRK-49F cells. Treatment with TGF- $\beta 1$ increased renal fibroblast viability by $\sim 1.3$-fold compared with vehicle-treated cells. VPA treatment decreased TGF- $\beta 1$-induced cell proliferation in a dose-dependent manner (Fig. 7A). Cell migration was also evaluated using a wound healing assay. TGF- $\beta 1$-treated NRK-49F cells exhibited increased migration compared with baseline or vehicle-treated cells. VPA treatment significantly decreased the TGF- $\beta 1$-induced increase in cell migration (Fig. 7B). These data suggest that VPA treatment regulates TGF- $\beta 1$-induced renal fibroblast viability and migration in vitro.
VPA suppresses TGF- $\beta 1$-induced fibronectin and Collal promoter enrichment at a H3Ac site in NRK-49F cells. Whether VPA regulates TGF- $\beta 1$-induced myofibroblast activation and ECM production was evaluated in NRK-49F cells. After 24-h stimulation with TGF- $\beta 1, \alpha-S M A$, fibronectin and type I collagen expression were significantly increased compared with the control. However, VPA caused a dose-dependent decrease in $\alpha$-SMA, fibronectin and type I collagen expression in TGF- 31 -induced cells (Fig. 8A).

Histone acetylation has an important role in chromatin remodeling and regulation of gene transcription. The effect of VPA on H3Ac and Fnl and Collal promoter enrichment at acetyl-histone H3 was also evaluated. Treatment with VPA for $1 \mathrm{~h}$ significantly increased $\mathrm{H} 3 \mathrm{Ac}$ at lysine 9 and 14 in a dose-dependent manner in NRK-49F cells however, TGF- $\beta 1$ 
A
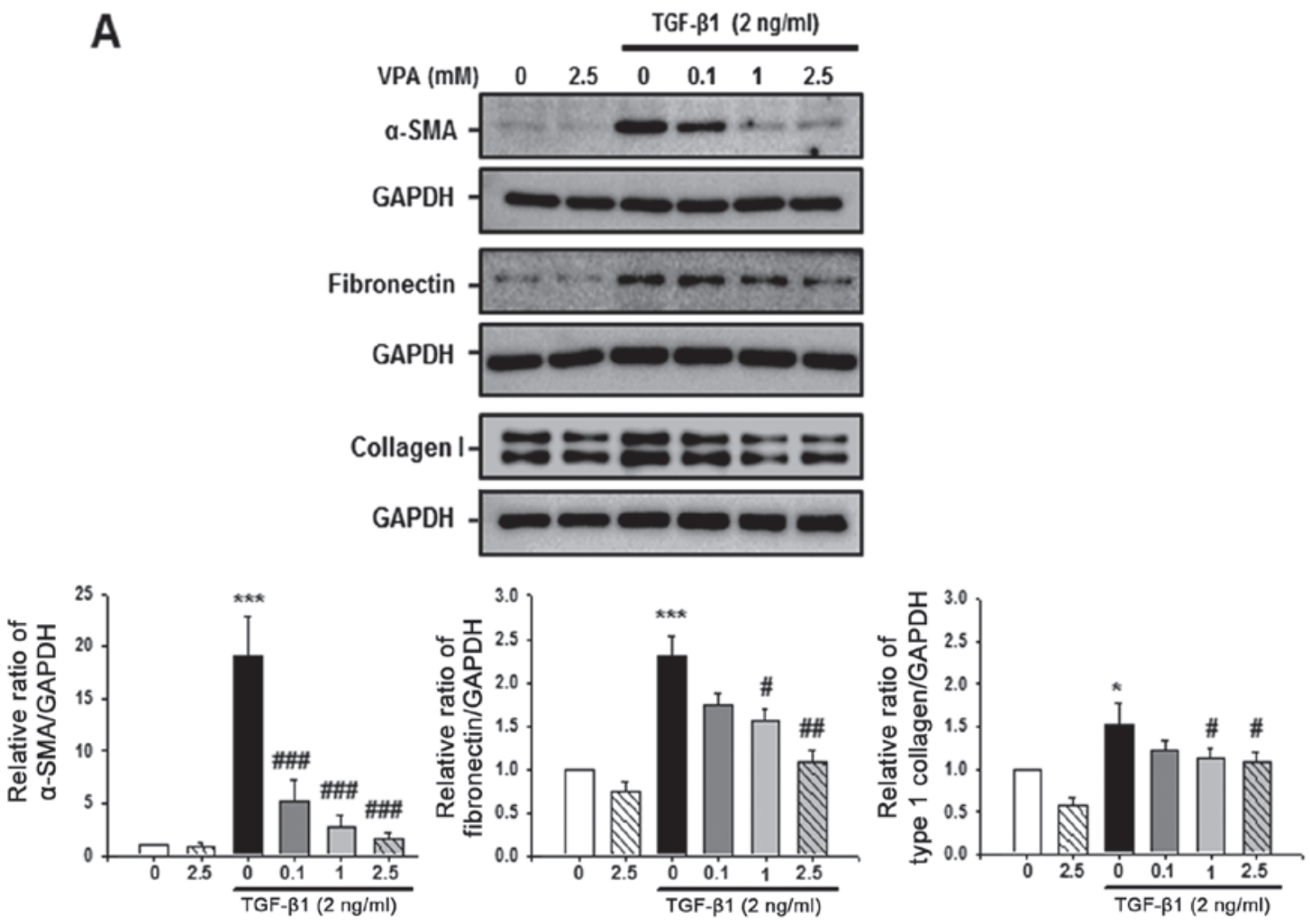

B

TGF- $\beta 1$ (2 ng/ml)
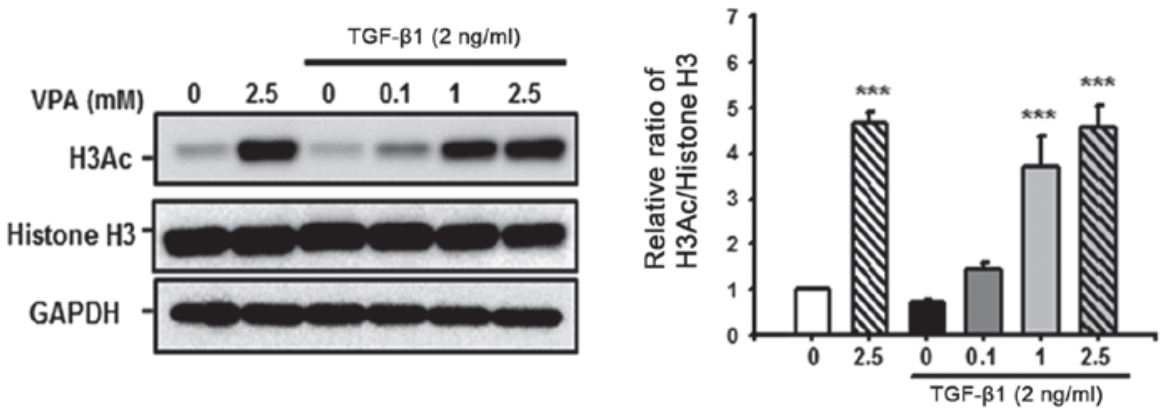

C

D
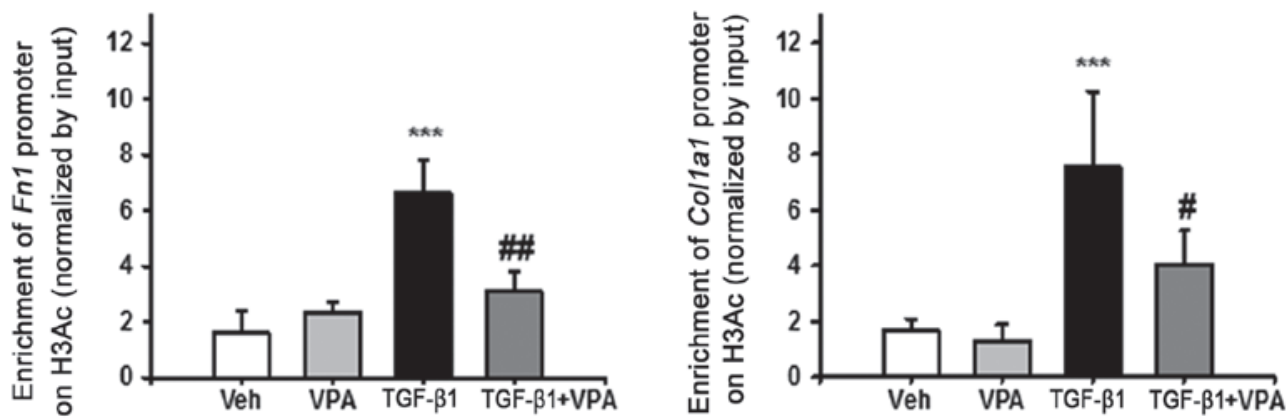

Figure 8. Effect of VPA on TGF- $\beta 1$-induced myofibroblast activation and extracellular matrix expression in NRK-49F cells. (A) A representative western blot analysis for $\alpha$-SMA, Fn1 and type I collagen expression in NRK-49F cells after $24 \mathrm{~h}$ TGF- $\beta 1$ treatment with or without VPA at indicated doses. Data from densitometric analyses are presented as the relative ratio of each protein to GAPDH. The relative ratio measured from Veh-treated NRK-49F cells is arbitrarily presented as 1 . Data are expressed as the mean $\pm \mathrm{SD}$ from three independent experiments. ${ }^{* * * *} \mathrm{P}<0.001$ vs. Veh or VPA; ${ }^{\#} \mathrm{P}<0.05 \mathrm{vs}$. TGF- $\beta 1 ;{ }^{\# \#} \mathrm{P}<0.01$ vs. TGF- $\beta 1 ;{ }^{\# \#} \mathrm{P}<0.001$ vs. TGF- $\beta 1$. (B) A representative western blot analysis for H3Ac at lysine 9 and 14 in NRK- $49 \mathrm{~F}$ cells after $1 \mathrm{~h}$ treatment with TGF- $\beta 1$ with or without VPA at the indicated doses. Data from densitometric analyses are presented as the relative ratio of each protein to histone H3. The relative ratio measured from Veh-treated NRK- $49 \mathrm{~F}$ cells is arbitrarily presented as 1 . Data are expressed as the mean $\pm \mathrm{SD}$. from three independent experiments. ${ }^{* * *} \mathrm{P}<0.001$ vs. Veh. Chromatin immunoprecipitation assay of (C and D) Fnl and Coll $\alpha 1$ promoter regions in NRK-49F cells after $1 \mathrm{~h}$ treatment of Veh or TGF- $\beta 1$ ( $2 \mathrm{ng} /$ $\mathrm{ml})$ with or without VPA $(2.5 \mathrm{mM})$. Immunoprecipitation was performed using acetyl-H3 antibody. Normal mouse IgG was used as a negative control and the PCR product of input chromatin (input 5\%) before immunoprecipitation was used as a positive control. Quantitative polymerase chain reaction was performed using specific primers for $F n 1$ and Collal promoter regions. All results were normalized to input levels. Data are expressed as the mean \pm SD from four independent experiments. ${ }^{* * *} \mathrm{P}<0.001$ vs. Veh or VPA ; $\mathrm{P}<0.05$ vs. TGF- $\beta 1 ;{ }^{\# \#} \mathrm{P}<0.01$ vs. TGF- $\beta 1$. TGF- $\beta 1$, transforming growth factor- $\beta 1$; VPA, valproic acid; $\alpha$-SMA, $\alpha$-smooth muscle actin; H3Ac, acetylated histone 3; Fn1, fibronectin 1; Coll $\alpha 1$, type I collagen $\alpha 1$; Veh, vehicle; SD, standard deviation. 
did not have an effect on histone $\mathrm{H} 3$ acetylation (Fig. 8B). Subsequently, ChIP assay was performed to address whether VPA-induced H3Ac was associated with ECM promoter enrichment in NRK-49F cells. Following H3Ac immunoprecipitation, Fnl and Coll $\alpha 1$ promoter enrichment on H3Ac was increased by treatment with TGF- $\beta 1$ compared with vehicle or VPA-treated NRK-49F cells. VPA significantly decreased the TGF- $\beta 1$-induced increase in $F n l$ and Coll $\alpha 1$ promoter enrichment (Fig. 8B and C). These data suggest that VPA promotes $\mathrm{H} 3$ acetylation at lysine 9 and 14, and regulates $F n 1$ and Coll $\alpha 1$ promoter enrichment at the acetyl-histone H3. Thus, VPA decreases the TGF- $\beta 1$-induced increase of Fnl and Coll $\alpha 1$ gene expression via histone modification at ECM promoters.

\section{Discussion}

Following tissue injury, inflammation and fibrosis are precisely regulated by the immune system for tissue repair. However, in most cases, the inflammatory response and myofibroblast activation stimulate an inappropriate pro-fibrotic response (22). Therefore, regulation of the inflammatory response and myofibroblast activation are potential targets for anti-fibrotic therapy. The results of the current study suggest that HDAC1 inhibition by VPA suppresses UUO-induced inflammation, myofibroblast activation and proliferation, and ECM deposition. VPA also inhibits TGF- $\beta 1$-induced renal fibroblast proliferation and migration, and ECM gene promoter enrichment at acetylated histone H3 in NRK-49F cells. These results suggest that VPA has therapeutic potential for preventing renal fibrosis by regulation of myofibroblast activation and ECM production.

Fibrosis is the final common pathological feature in chronic inflammatory disease and is characterized by accumulation of ECM proteins, including fibronectin and collagens (23). There are various factors that contribute to the development of fibrotic disease. Among them, activation of myofibroblasts is a hallmark of fibrogenesis (23). Although the origin of myofibroblasts is controversial, activation of local fibroblasts, phenotypic changes from epithelial, endothelial, pericyte and bone marrow-derived fibroblasts have been suggested as origins of myofibroblasts in fibrotic tissues (24). The results of the current study demonstrated that VPA treatment inhibited the UUO-induced myofibroblast infiltration and proliferation based on double immunofluorescence staining for $\alpha$-SMA and Ki-67. Furthermore, VPA decreased the TGF- $\beta 1$-induced increase in fibroblast activation, proliferation, and migration in NRK-49F cells. Therefore, the in vivo and in vitro data suggest that regulation of myofibroblast activation is an important therapeutic target for progressive renal fibrosis.

In the UUO model, there is robust infiltration of inflammatory cells around tubulointerstitial areas. Cell adhesion molecules, chemokines and their receptors and cytokines regulate the inflammatory cells transmigration via the vascular endothelium and differentiation into macrophages and dendritic cells (25). The in vivo data of the current study indicated that there was increased infiltration of F4/80(+) macrophages accompanied by increased expression of ICAM-1 in the interstitial area, and increased expression of MCP-1 at the dilated tubules following ureteral obstruction. VPA treatment ameliorated UUO-induced renal inflammation via regulation of inflammatory cell infiltration and proinflammatory cytokine expression. Therefore, regulation of the renal inflammatory process is one suggested protective mechanism for VPA in UUO-induced renal injury.

TGF- $\beta 1$ is an important mediator of renal fibrosis, and the downstream Smad signaling pathway is crucial in regulating TGF- $\beta 1$-induced fibrosis $(7,26)$. Following activation of TGF- $\beta 1$ signaling, the Smad effector proteins have distinct roles in renal fibrosis (21). The in vivo data in the present study demonstrate that VPA decreases the UUO-induced increase in Smad2 and Smad3 phosphorylation, which has a profibrotic role in kidney injury. By contrast, Smad7, an inhibitory Smad, is decreased following ureteral obstruction. VPA restores the expression of Smad7, which reduces the activation of TGF- $\beta 1 /$ Smad signaling. These results suggest that VPA could potentially alter TGF- $\beta 1 / \mathrm{Smad}$ signaling pathway in renal fibrosis.

The final fibrotic process involves the accumulation and dysregulated remodeling of ECM proteins (27). According to Seet et al (28), VPA suppresses type I collagen synthesis via the TGF- $\beta 1$ regulatory pathway in conjunctival fibroblasts and mouse glaucoma model. The results of the current study also demonstrate that VPA decreases the TGF- $\beta 1$-induced increase in ECM, fibronectin and type I collagen expression in NRK-49F cells and UUO kidneys. In contrast to the study of Seet et al (28), ChIP results from the current study indicated that VPA increases H3Ac and regulates enrichment of $F n 1$ and Coll $\alpha 1$ promoter at acetylated histone H3. Thus, VPA may inhibit TGF- $\beta 1$-induced increases in ECM gene expression via increased $\mathrm{H} 3$ acetylation at lysine 9 and 14 .

Tissue inhibitor of metalloproteinases have important roles in inhibiting ECM proteolysis and controlling ECM turnover, which is dependent on metalloproteinase inhibition (29). However, in the current study, the focus was on H3Ac and regulation of transcription from ECM promoters via histone acetylation. This is a limitation of the present study and further studies are required to determine the effect of VPA on regulation of metalloproteinase activities in kidney fibrosis. Another limitation is the known toxicity of VPA when used in the treatment of patients with epilepsy, including side-effects such as cerebral edema (30) and hyperammonemic encephalopathy (31), hepatotoxicity (32) and electrolyte imbalances (33). These toxicities are usually associated with overdose of VPA. Therefore, caution would be required if using VPA for the treatment of renal fibrosis.

In conclusion, the findings of the current study indicate that VPA may have a protective effect against UUO-induced tubulointerstitial fibrosis via regulation of inflammation, renal fibroblast activation and TGF- $\beta 1 / \mathrm{Smad}$ signaling. In addition, the data suggest that VPA decreased UUO-induced ECM production via regulation of chromatin remodeling and $F n 1$ and Collal promoter enrichment at the H3Ac sites. Therefore, targeting HDAC1 inhibition in renal fibrosis may be a novel therapeutic approach for preventing and treating CKD.

\section{Acknowledgements}

We thank Kieu Thi Thu Trang for the excellent technical assistance. This study was supported by the National Research Foundation of Korea (NRF) funded by the Korean 
government (NRF-2015R1D1A3A03015653, to K.P.K and NRF-2014R1A1A4A01003832, to S.K.P) and by research funds of Chonbuk National University in 2014 (to K.P.K).

\section{References}

1. Eckardt KU, Coresh J, Devuyst O, Johnson RJ, Köttgen A, Levey AS and Levin A: Evolving importance of kidney disease: From subspecialty to global health burden. Lancet 382: 158-169, 2013.

2. Sharif MU, Elsayed ME and Stack AG: The global nephrology workforce: Emerging threats and potential solutions! Clin Kidney J 9: 11-22, 2016.

3. Couser WG, Remuzzi G, Mendis S and Tonelli M: The contribution of chronic kidney disease to the global burden of major noncommunicable diseases. Kidney Int 80: 1258-1270, 2011.

4. No authors listed: The global issue of kidney disease. Lancet 382: 101,2013

5. Braun L, Sood V, Hogue S, Lieberman B and Copley-Merriman C: High burden and unmet patient needs in chronic kidney disease. Int J Nephrol Renovasc Dis 5: 151-163, 2012.

6. Liu Y: Cellular and molecular mechanisms of renal fibrosis. Nat Rev Nephrol 7: 684-696, 2011.

7. He W and Dai C: Key Fibrogenic Signaling. Curr Pathobiol Rep 3: 183-192, 2015.

8. Xia J, He LQ and Su X: Interventional mechanisms of herbs or herbal extracts on renal interstitial fibrosis. J Integr Med 14: $165-173,2016$.

9. Morikawa M, Derynck R and Miyazono K: TGF- $\beta$ and the TGF- $\beta$ family: Context-dependent roles in cell and tissue physiology. Cold Spring Harb Perspect Biol 8: a021873, 2016.

10. Kim D, Lee AS, Jung YJ, Yang KH, Lee S, Park SK, Kim W and Kang KP: Tamoxifen ameliorates renal tubulointerstitial fibrosis by modulation of estrogen receptor $\alpha$-mediated transforming growth factor- $\beta 1 /$ Smad signaling pathway. Nephrol Dial Transplant 29: 2043-2053, 2014.

11. Chateauvieux S, Morceau F, Dicato M and Diederich $M$ Molecular and therapeutic potential and toxicity of valproic acid. J Biomed Biotechnol 2010: 479364, 2010.

12. Blaheta RA, Michaelis M, Driever PH and Cinatl J Jr: Evolving anticancer drug valproic acid: Insights into the mechanism and clinical studies. Med Res Rev 25: 383-397, 2005.

13. Göttlicher M, Minucci S, Zhu P, Krämer OH, Schimpf A, Giavara S, Sleeman JP, Lo Coco F, Nervi C, Pelicci PG, et al: Valproic acid defines a novel class of HDAC inhibitors inducing differentiation of transformed cells. EMBO J 20: 6969-6978, 2001.

14. Liu N and Zhuang S: Treatment of chronic kidney diseases with histone deacetylase inhibitors. Front Physiol 6: 121, 2015.

15. Khan S, Jena G and Tikoo K: Sodium valproate ameliorates diabetes-induced fibrosis and renal damage by the inhibition of histone deacetylases in diabetic rat. Exp Mol Pathol 98: 230-239, 2015.

16. Van Beneden K, Geers C, Pauwels M, Mannaerts I, Verbeelen D, van Grunsven LA and Van den Branden C: Valproic acid attenuates proteinuria and kidney injury. J Am Soc Nephrol 22 $1863-1875,2011$
17. Zheng Q, Liu W, Liu Z, Zhao H, Han X and Zhao M: Valproic acid protects septic mice from renal injury by reducing the inflammatory response. J Surg Res 192: 163-169, 2014.

18. Chevalier RL, Forbes MS and Thornhill BA: Ureteral obstruction as a model of renal interstitial fibrosis and obstructive nephropathy. Kidney Int 75: 1145-1152, 2009.

19. Meng XM, Nikolic-Paterson DJ and Lan HY: Inflammatory processes in renal fibrosis. Nat Rev Nephrol 10: 493-503, 2014.

20. Kovacs EJ and DiPietro LA: Fibrogenic cytokines and connective tissue production. FASEB J 8: 854-861, 1994.

21. Meng XM, Nikolic-Paterson DJ and Lan HY: TGF- $\beta$ : The master regulator of fibrosis. Nat Rev Nephrol 12: 325-338, 2016.

22. Lupher ML Jr and Gallatin WM: Regulation of fibrosis by the immune system. Adv Immunol 89: 245-288, 2006.

23. Wynn TA and Ramalingam TR: Mechanisms of fibrosis: Therapeutic translation for fibrotic disease. Nat Med 18: 1028-1040, 2012.

24. Grande MT and López-Novoa JM: Fibroblast activation and myofibroblast generation in obstructive nephropathy. Nat Rev Nephrol 5: 319-328, 2009.

25. Alikhan MA and Ricardo SD: Mononuclear phagocyte system in kidney disease and repair. Nephrology (Carlton) 18: 81-91, 2013.

26. Boor P, Ostendorf T and Floege J: Renal fibrosis: Novel insights into mechanisms and therapeutic targets. Nat Rev Nephrol 6: 643-656, 2010.

27. Genovese F, Manresa AA, Leeming DJ, Karsdal MA and Boor P: The extracellular matrix in the kidney: A source of novel non-invasive biomarkers of kidney fibrosis? Fibrogenesis Tissue Repair 7: 4, 2014.

28. Seet LF, Toh LZ, Finger SN, Chu SW, Stefanovic B and Wong TT: Valproic acid suppresses collagen by selective regulation of Smads in conjunctival fibrosis. J Mol Med (Berl) 94: 321-334, 2016.

29. Arpino V, Brock M and Gill SE: The role of TIMPs in regulation of extracellular matrix proteolysis. Matrix Biol 44-46: 247-254, 2015.

30. Dupuis RE, Lichtman SN and Pollack GM: Acute valproic acid overdose. Clinical course and pharmacokinetic disposition of valproic acid and metabolites. Drug Saf 5: 65-71, 1990.

31. Dealberto MJ: Valproate-induced hyperammonaemic encephalopathy: Review of 14 cases in the psychiatric setting. Int Clin Psychopharmacol 22: 330-337, 2007.

32. Bryant AE III and Dreifuss FE: Valproic acid hepatic fatalities. III. U.S. experience since 1986. Neurology 46: 465-469, 1996.

33. Zaki EL and Springate JE: Renal injury from valproic acid: Case report and literature review. Pediatr Neurol 27: 318-319, 2002. 